\title{
Deficiência física e envelhecimento: estudo das representações sociais de idosos sob reabilitação fisioterápica
}

\author{
Discapacidad y enuejecimiento: estudio de las representaciones \\ sociales de los ancianos de la rehabilitación de terapia física \\ Disability and figing: Study of Social Representations \\ of the Elderly from Physical Therapy Rehabilitation
}

\author{
Taciana Duarte de Queiroz Brito, Ana Raquel de Oliveira* \\ Universidade Federal da Paraiba \\ Maria do Carmo Eulálio \\ Universidade Estadual da Paraíba
}

Doi: dx.doi.org/10.12804/apl33.01.2015.09

\section{Resumo}

Este estudo teve como objetivo verificar as Representações Sociais de idosos acerca da deficiência física. Participaram quinze pessoas idosas, de ambos os sexos, com média de idade 70.6 anos, que estavam sendo submetidos a tratamento fisioterápico em uma Clínica de Fisioterapia, em Campina Grande - PB. Utilizou-se como instrumento um questionário sociodemográfico e a foto-linguagem com a técnica de Associação Livre de Palavras. O material coletado pela entrevista foi categorizado pela análise de conteúdo temática. Verificou-se, entre os idosos, uma representação da deficiência física em três categorias: os sentimentos despertados; a importância da família; e uma perspectiva futura positiva. Conclui-se que a deficiência, para estes idosos, representa um período de transição em que eles acreditam na dinâmica da reabilitação. Este estado emocional positivo, deve ajudar no estado geral do idoso, determinando condição essencial para a reabilitação.

Palavras-chave: Idoso, deficiência física, representações sociais

\section{Resumen}

Este estudio tuvo como objetivo verificar las representaciones sociales de ancianos sobre la discapacidad física. Participaron quince ancianos, de ambos sexos, con una edad promedio de 70.6 años, que fueron sometidos a tratamiento de fisioterapia en una clínica de Fisioterapia en Campina Grande - PB. Se utilizó como instrumento un cuestionario sociodemográfico y la técnica del foto-lenguaje con la asociación libre de palabras. El material recogido por medio de entrevistas se clasificó por

* Taciana Duarte de Queiroz Brito, Universidade Federal da Paraíba; Ana Raquel de Oliveira, Universidade Federal da Paraíba; Maria do Carmo Eulálio, Universidade Estadual da Paraíba.

A correspondência relacionada com este artigo deve ser direcionada a: Taciana Duarte de Queiroz Brito, Universidade Federal da Paraíba. Correio eletrônico: tacydq@hotmail.com

Para citar este artigo: De Queiroz Brito, D. T., De Oliveira, A. R., \& Eulálio, M. (2015). Deficiência física e envelhecimento: estudo das representações sociais de idosos sob reabilitação fisioterápica. Avances en Psicología Latinoamericana 33(1), 121-133. doi: dx.doi.org/10.12804/apl33.01.2015.09 
el análisis de contenido temático. Se encontró entre los ancianos una representación de la discapacidad física en tres categorías: los sentimientos que despierta, la importancia de la familia y una perspectiva futura positiva. Se llegó a la conclusión de que la discapacidad para estos ancianos representa un período de transición en el que ellos creen en la dinámica de la rehabilitación. Este estado emocional positivo debe ayudar en el estado general del anciano, lo que determina una condición esencial para la rehabilitación.

Palabras clave: Anciano, discapacidad física, representaciones sociales

\section{fbstract}

This study persons aimed to verify the Social Representations of elderly persons about physical disability. Participants were 15 elderly persons, of both sexes, with mean age 70.6 years, who were undergoing physiotherapy treatment at a Physiotherapy Clinic, in Campina Grande - PB. We used as instruments a sociodemographic questionnaire and photo-language with the free association technique. The material collected by interviews was categorized by thematic content analysis. It was found among the elderly, a representation of disability in three categories: the feelings aroused, the importance of family, and a positive future outlook. It is concluded that disability for these elderly persons represents a transition period in which they believe in the dynamics of rehabilitation. This positive emotional state, should help the overall condition of the elderly, determining a precondition for rehabilitation.

Keywords: Senior, physical disability, social representations

O envelhecimento populacional é um fenômeno mundial. Segundo projeções populacionais da Organização das Nações Unidas (ONU, 2010), a esperança de vida média chegará a 76 anos entre 2045-2050 e de acordo com a Organização Mundial de Saúde (OMS; 2010), em países menos des- envolvidos, poderá chegar a 74 anos e, nos mais desenvolvidos a previsão é de 83 anos.

Dados do Instituto Brasileiro de Geografia e Estatística (IBGE; 2010) apontam que a população idosa corresponde a $10.8 \%$ da população brasileira. O Banco Mundial (2011), por sua vez, estima que nos próximos 40 anos a taxa de crescimento da população idosa corresponderá a $3.2 \%$ ao ano (enquanto a população total crescerá a uma taxa de $0.3 \%$ ) e atingirá 64 milhões de habitantes em 2050.

Segundo o Estatuto do Idoso (Brasil, 2003), são consideradas idosas as pessoas com idade igual ou superior a 60 anos. A OMS (1984) considera o idoso, sob o ponto de vista cronológico, como aquele indivíduo que possui 65 anos ou mais de idade em países desenvolvidos, enquanto que, em países em desenvolvimento, como é o caso do Brasil, prevalece à idade de 60 anos ou mais. Dentre o contingente da população brasileira, que se encontra na etapa da velhice, tem-se um grande número de pessoas com deficiência física (IBGE, 2010). O levantamento censitário realizado pelo referido instituto, investigou a prevalência das deficiências: visual, auditiva, motora e intelectual. Os dados indicaram que, em crianças de 0 a 14 anos de idade, $7.5 \%$ apresentaram pelo menos um tipo de deficiência. $\mathrm{Na}$ população de 15 a 64 anos, a prevalência de pelo menos uma das deficiências foi $23.9 \%$, enquanto que na população com 65 anos ou mais, a prevalência atingiu mais da metade $(67.7 \%)$.

Esse aumento proporcional, da prevalência de deficiência em relação à idade, pode ser atribuído às peculiaridades decorrentes do próprio processo de envelhecimento, em que a complexa interação entre vários processos neurobiológicos podem tornar o idoso suscetível à perda gradual da acuidade visual, auditiva e da capacidade motora (Fjell et al., 2013).

Desse modo, a qualidade de vida dessas pessoas tem interessado aos pesquisadores e profissionais que atuam diretamente com questões relacionadas às deficiências. Isto por que os avanços da medicina e os progressos sociais, relativos à consideração 
dos direitos de pessoas com deficiência, têm aumentado às chances de essas pessoas viverem por mais tempo (Resende \& Neri, 2005).

Resende e Neri (2005) asseveram que a deficiência pode ser socialmente entendida como o resultado do descompasso entre: as condições do indivíduo afetado por uma limitação funcional, suas expectativas quanto à execução das atividades básicas e instrumentais de vida diária, e a escassez ou a inadequação de condições instrumentais e sociais que lhe proporcionem funcionar adequadamente, mantendo a autonomia e a autoestima.

Destarte, várias áreas de atuação podem propiciar intervenção adequada, a exemplo da reabilitação física oferecida pela fisioterapia, cuja meta principal é ajudar o indivíduo a atingir a maior independência funcional possível, considerando suas potencialidades e limitações. O tratamento fisioterápico, especificamente assume papel importante em relação à melhora dos sintomas e à restauração da função, podendo conferir uma maior qualidade de vida aos idosos (Barduzzi et al., 2013).

Deste modo, evidencia-se a necessidade da garantia da qualidade de vida e bem-estar desse contingente populacional que convive com a redução de suas capacidades funcionais e requer cuidados especiais (Batista, Jaccoud, Aquino, L., \& El-Moor, 2008). Logo, a reflexão sobre o tema envelhecimento e deficiência física conduz ao entendimento de que a abordagem social desta temática poderá contribuir à produção do conhecimento e de mecanismos de intervenção direcionados a este segmento social.

\section{Envelhecimento, deficiência e dependência}

O envelhecimento pode ser compreendido como um processo multidimensional que envolve uma série de fatores, tanto biológicos quanto psicológicos, sociais e culturais.

Na perspectiva biológica, a velhice é demarcada pela "involução morfofuncional que afeta todos os sistemas fisiológicos principais, de forma variável" (Moraes, Moraes, \& Lima, 2010, p. 67).

Do ponto de vista psicológico, emergiram várias teorias, dentre as quais o paradigma do desenvolvimento ao longo de toda a vida (lifespan) (Baltes, 1987; Baltes \& Baltes, 1990), o qual tem contribuído a mudanças de concepções acerca da velhice. Esta perspectiva concebe o envelhecimento como um processo multideterminado e heterogêneo, que pode ser categorizado em normal, patológico e ótimo ou saudável (Baltes, 1987).

A partir da década de 90, emergiram as teorias psicológicas da seletividade sócio-emocional (Carstensen, 1992) e da dependência aprendida (Baltes, 1996). A primeira procura explicar o declínio nas interações sociais e as mudanças no comportamento emocional dos idosos. Assim, preconiza que, a redução na amplitude da rede de relações sociais e na participação social na velhice, reflete a redistribuição de recursos socioemocionais pelos idosos, dado que sua perspectiva de tempo futuro parece cada vez mais limitada.

Para compreender a teoria da dependência aprendida, é necessário resgatar a literatura gerontológica a respeito do conceito da dependência, compreendida como a incapacidade de a pessoa funcionar satisfatoriamente, sem ajuda, devido a limitações físico-funcionais, cognitivas ou a uma combinação dessas duas condições. Acreditava-se que a dependência era uma condição obrigatória e unidimensional na velhice. No entanto, essa assertiva não condiz com a realidade, posto que a dependência não só é uma condição multifacetada, como também é determinada pela interação de múltiplas variáveis (Baltes, 1996).

Assim sendo, a teoria da dependência aprendida, agrega novos elementos à análise da dependência na velhice. Um primeiro elemento é a noção compartilhada pelos teóricos da aprendizagem social, de que a dependência não é uma condição específica da velhice, mas é um fenômeno que ocorre ao longo de todo o ciclo vital, embora de maneiras diferentes (Neri, 2006). 
O surgimento de novos paradigmas para a senescência e para o processo de envelhecimento, segundo Neri (2004), contribuiu a um maior cuidado em relação aos idosos na sociedade contemporânea. No entanto, a "velhice" ainda é definida como uma época em que o indivíduo passa por muitas perdas, como a diminuição da resistência a doenças, mudanças corporais e a redução da força e da agilidade, existindo um estereótipo do idoso como um ser incapaz e improdutivo.

Para Jede e Spuldaro (2009) as doenças que naturalmente podem vir a acometer os idosos, fazem emergir um fator emocional de regressão, acentuando os sentimentos de fragilidade, dependência e insegurança. Com isso, repercussões psíquicas são inevitáveis, acarretando em preocupações, angústias, medos, alterações na autoimagem e dependência.

No entanto, a teoria da dependência aprendida, preconiza que o significado da dependência para os indivíduos adultos e idosos e para a sua rede de relações sociais mais próximas, pode produzir maior ou menor tolerância e aceitação, e proporcionar melhor ou pior suporte instrumental, informativo, material e afetivo. Postula ainda que, a dependência na velhice reflete as condições do sistema microssocial no qual o idoso está inserido, condições estas que abarcam o seu sistema de crenças e de repertórios comportamentais (Neri, 2006).

Para Baltes (1996), a dependência significa perdas, uma vez que dificulta o engajamento em ações que promovem funcionalidade física e psicossocial, mas também significa ganhos, pois auxilia as pessoas a obter atenção, contato social e controle passivo. Além disso, ajuda a preservar, canalizar e otimizar energias para outros objetivos. Nesta concepção a dependência pode ser uma estratégia adaptativa.

Segundo Resende e Neri (2009), envelhecer bem requer ajustamento pessoal e social, o que pode vir a ser comprometido devido às condições deficitárias de saúde e educação ao longo do curso de vida. Caso o indivíduo tenha alguma deficiência física congênita ou adquirida antes da velhice, suas condições de desenvolver-se e envelhecer com sucesso poderão sofrer prejuízo, a depender da extensão e da natureza da deficiência, dos recursos de apoio que o ambiente sociocultural oferece e dos recursos psicológicos de que dispõem.

As atitudes e as crenças, que o indivíduo detém sobre si mesmo e sobre suas relações com o mundo, constituem um aspecto essencial da adaptação do self. São formadas a partir da experiência direta e da interação social, desempenhando papel central nos esforços de ajustamento pessoal (Resende \& Neri, 2009).

Portanto, se faz necessário conhecer quais as concepções dos idosos com deficiência física a respeito de sua condição, tema ainda pouco explorado pelo campo de estudo da Gerontologia. Para embasar este trabalho buscou-se aporte teórico na Psicologia Social, sob a ótica psicossocial da Teoria das Representações Sociais (TRS; Moscovici, 2009) a qual fundamenta os conhecimentos gerados no dia a dia.

\section{Representações sociais e envelhecimento}

As representações sociais são um fenômeno típico da sociedade moderna (Moscovici, 2009). Representam o conjunto de conceitos, afirmações e explicações que são tecidas diariamente, a partir da comunicação e da cooperação entre indivíduos e grupos. O interesse dessa teoria reside em apreender como um novo conhecimento científico se difunde e é apropriado por diferentes grupos sociais. Deste modo, Saraiva e Coutinho (2012) asseveram que estudar representação social implica em focalizar como o ser humano compreende o mundo e não como ele se comporta.

De acordo com Neri (1991), o significado da velhice é construído a partir da percepção pessoal, por intermédio dos valores e experiências, crenças e atitudes que cada um internaliza, bem como da história interacional do indivíduo com eventos contextuais de natureza reforçadora, avaliativa e 
diretiva. Segundo Araújo Sá e Amaral, (2011), as representações sociais acerca dos idosos acabam por provocar uma não identificação, por parte dos mesmos, com essa etapa da vida, já que grande parte das pessoas com 60 anos ou mais não se vê dessa forma; o idoso é sempre o outro.

Resende e Rabelo (2004) investigaram as concepções de um grupo de idosos, que frequentavam uma associação de paraplégicos, acerca da velhice e deficiência física. Apreenderam que, de modo geral, houve uma dificuldade na aceitação da velhice pessoal, pois evidenciaram que não poderiam ainda ser considerados velhos uma vez que não estavam doentes e dependentes. Isto indica que a palavra "velho" ainda carrega muitas conotações negativas numa sociedade onde se supervaloriza adjetivos relacionados à juventude. Já com relação à deficiência física, os idosos demonstraram que este não é um fator ameaçador, pois acreditam estar bem adaptados à sua condição de viver com uma deficiência.

Albrecht e Devlieger (1999) investigaram as atitudes de 153 pessoas com deficiência, $54.3 \%$ dos participantes responderam ter excelente ou ótima qualidade de vida, o que levou a considerar uma atitude positiva frente à deficiência física. Dessa forma, depreende-se que as atitudes das pessoas com deficiência estão relacionadas à percepção sobre sua qualidade de vida, entendida como noção geral de bem-estar.

Estes dados reforçam a tese de que a presença de algum grau de incapacidade para o desempenho de atividades básicas e instrumentais de vida diária não significa necessariamente impedimento para a continuidade do funcionamento cognitivo e emocional. Isto por que é possível preservar a autonomia que se manifesta por meio da capacidade de tomar decisões. Ao manter a integridade cognitiva e dos mecanismos de autorregulação do self, adultos e idosos com deficiência física podem ativar mecanismos de adaptação para enfrentar perdas em funcionalidade (Neri, 2000).
Acredita-se que, o conhecimento sobre o que os idosos pensam acerca da convivência com a deficiência física, propicie uma melhor compreensão dos aspectos singulares do seu envelhecimento. Dessa forma, este estudo poderá colaborar ao desenvolvimento de ações de saúde no âmbito multi, inter ou transdisciplinar que contemplem o saber desta população acerca de sua própria condição.

Tendo por base um enfoque psicossociológico, o objetivo deste estudo foi conhecer as representações sociais que emergiram dos discursos das pessoas idosas sobre a deficiência física.

\section{Método}

Esta pesquisa, a priori, iniciou-se após receber parecer favorável do Comitê de Ética em pesquisa, do Centro de Ciências Biológicas em Saúde, da UEPB, que regulamenta experimentação científica em seres humanos, por estar de acordo com a Resolução 466/12 do Conselho Nacional de Saúde/ Ministério da Saúde do Brasil (Brasil, 2012).

\section{Tipo de estudo}

Trata-se de uma pesquisa exploratória, com abordagem qualitativa.

\section{Caracterização dos participantes}

Com relação à amostra, não houve critério de seleção quanto à questão de gênero, devido a quantidade de idosos que frequentavam a clínica no período de coleta, sendo realizada aleatoriamente.

Participaram 15 pessoas idosas, com idade variando entre 61 e 81 anos $(M=70,6)$, sendo homens $(60 \%)$ e mulheres $(40 \%)$, com deficiências físicas, a saber: traumas ortopédicos $(60 \%)$ e neurológicos $(40 \%)$. Todos estavam submetidos a tratamento fisioterápico em uma Clínica de Fisioterapia, situada em uma universidade estadual do interior da Paraíba-PB. Em relação ao estado 
civil, houve predominância dos casados (46\%), sobre os solteiros (27\%) e viúvos (27\%). Quanto ao grau de escolarização, $40 \%$ possuíam o ensino fundamental incompleto, e $20 \%$ ensino superior completo, sendo a mesma parcela $(20 \%)$ para os não alfabetizados.

As ocupações, anteriores aos traumas, declaradas pelos idosos eram: agricultura (20\%), "do lar" (13.3\%), sapateiro, técnico em tratamento de água, eletricista, consertador de máquinas, mecânico de automóveis, contador, costureira, doméstica, professor e fotógrafo. A maioria estava aposentada ( $74 \%$ ), ou era pensionista (13\%), ou não recebiam nenhum benefício (13\%), mas estes estavam em fase de tramitação da papelada no órgão competente, para fins de benefícios de aposentadoria e/ou pensão.

No tocante à religião, $80 \%$ declararam-se católicos, $13 \%$ protestantes e $7 \%$ espíritas. Com relação à moradia, a maioria dos entrevistados residia em casa própria ( $87 \%$ ) e a minoria em casa alugada $(13 \%)$. E quanto à companhia na moradia, a maior parte deles vivia com o (a) seu (sua) companheiro (a) e com os filhos ( $47 \%$ ), ou só com o filho (26\%), alguns moravam com outros familiares $(20 \%)$, ou sozinho (a) (7\%).

\section{Instrumentos}

Foram utilizados um questionário sociodemográfico e a técnica de associação livre de palavras, com o uso da foto-linguagem. O questionário sociodemográfico possuía questões referentes à idade, estado civil, escolaridade, ocupação anterior e atual, religião, tipo de moradia e tipo de deficiência, entre outras, visando caracterizar o perfil da amostra.

A associação livre de palavras é uma técnica bastante utilizada na Psicologia Social, sobretudo, quando se trabalha com o suporte teórico das RS, uma vez que possibilita acesso aos conteúdos periféricos e latentes (Castanha \& Araújo, 2006; Nóbrega \& Coutinho, 2003).
Explorar o recurso da fotografia como instrumento de pesquisa, pode ser bastante útil e contribuir significativamente para a investigação científica na Psicologia, apesar de ser um recurso ainda pouco utilizado nas pesquisas desta área (Justo \& Vasconcelos, 2009). De acordo com Neiva-Silva e Koller (2002), o objetivo principal, ao se trabalhar com a fotografia, é a atribuição de significado à imagem.

Assim, para auxiliar na apreensão das concepções dos idosos acerca da deficiência física, foi utilizado como estímulo indutor a foto-linguagem que consistiu em mostrar 5 fotografias (cada uma foi separada e colada em uma folha de ofício A4), que representavam pessoas com algum tipo de deficiência, e era solicitado aos participantes que fossem escolhidas apenas três, aquelas que mais se identificavam, para que pudessem evocar sentimentos relacionados à sua própria problemática.

Com relação ao conteúdo das fotos, a 1 continha uma senhora sorrindo que estava sentada em uma rede, com um apoio (muleta) no braço; a 2 , correspondia a um senhor, que estava sorrindo, em pé e apoiando-se também em uma muleta. $\mathrm{Na}$ foto 3, apresentava uma professora de dança em pé, dançando com as mãos dadas à uma menina, que estava em uma cadeira de rodas, o cenário era um palco e estavam durante uma apresentação de dança; já na 4, aparecia um homem em uma cadeira de rodas conversando com uma mulher que estava em pé à sua frente; e por último, a foto 5 , mostrava um homem na cadeira de rodas sendo empurrado por uma mulher, e uma outra segurando a sua mão ao seu lado.

\section{Procedimentos}

Inicialmente, os participantes foram recrutados na clínica escola de fisioterapia, sendo esclarecidos quanto aos objetivos do estudo, bem como o caráter voluntário da participação, sigilo e confidencialidade das informações. Em seguida, foram convidados 
a participar do estudo e, com os que aceitaram, foi combinado dia e horário para deslocarem-se até a clínica escola de fisioterapia, onde a pesquisa foi realizada com cada sujeito individualmente.

Os participantes assinaram o Termo de Consentimento Livre e Esclarecido (TCLE) e em seguida, era explicado e aplicado o questionário sóciodemográfico. Eram lidas as questões deste pela pesquisadora, e após os participantes responderem, a mesma marcava suas respostas.

Posteriormente à aplicação do questionário, solicitou-se a cada um dos participantes que olhassem as fotos e escolhessem as três mais significativas para si. Depois foram indagados sobre o motivo da escolha e falaram livremente acerca das três fotos elencadas por eles. Todos os discursos dos entrevistados foram gravados em um aparelho áudiocassete, mediante a permissão do mesmo (de acordo com o TCLE), justificando-se, assim, a importância da cópia fidedigna do conteúdo relatado.

Os relatos dos participantes foram apresentados da seguinte forma: os idosos eram identificados pela letra "E" de entrevistado e por um número sequencial (exemplo: 1, 2, 3, 4, .., 9...). As letras " $F$ " e " $M$ " corresponderam ao sexo feminino e masculino, respectivamente; e a letra “ $a$ ”, serviu para caracterizar a idade do participante.

\section{Análise de dados}

Os dados referentes à foto-linguagem foram transcritos na íntegra, lidos em profundidade, categorizados por tema e analisados os seus conteúdos. As falas evocadas por meio da visualização das fotografias foram analisadas pela técnica da análise de conteúdo temática (Bardin, 2008). Esta técnica aplica-se à análise de textos escritos, orais, imagéticos ou gestuais, permitindo uma compreensão das comunicações no seu sentido e atribuindo-lhes significados explícitos e/ou ocultos (Coutinho, Estevam, Araújo, \& Araújo, 2011).

\section{Resultados e Discussão}

$\mathrm{Na}$ tentativa de apreender o significado da deficiência física para os idosos, foram usados como estímulos indutores, cinco fotos que representavam situações em que se encontravam pessoas com deficiência física. As categorias de análise foram definidas considerando-se a literatura (Araújo et al., 2011; Baltes, 1996; Neri, 2006; Neri \& Jorge, 2006), bem como o conteúdo evocado a partir da exibição das fotos.

Para fins de análise, foram consideradas três categorias: sentimentos positivos despertados; importância da família; e perspectiva futura positiva. Apresentam-se, a seguir, as categorias exemplificadas com verbalizações dos próprios idosos.

\section{Sentimentos positivos despertados}

Esta categoria refere-se aos sentimentos positivos que foram despertados mediante a visualização das imagens. De acordo com os relatos dos idosos, a escolha da foto 1, foi motivada por representar "alegria, felicidade, exemplo de vida, ter uma aparência bonita", pois muitos entrevistados falavam que "pela pessoa da foto estar sorrindo e feliz, não parecia ser deficiente".

Observou-se que, alguns entrevistados, lembravam-se de seus familiares que são também deficientes, mas não falavam de si mesmos, parecendo assim, a escolha distanciada de suas situações atuais ou uma negação destas.

Isso pode ser observado nas representações sociais emergidas nas falas a seguir:

"Ela tá alegre, bem sorridente, bonita, em vez de ser uma pessoa deficiente, tá tão feliz" (E 9, F, 67 a).

"Achei parecida com minha mãe, é bonita, é legal pra ser deficiente" (E 4, M, 63 a).

A partir dos discursos, percebeu-se que a imagem revelou a surpresa dos participantes em relação ao fato de a pessoa estar com boa aparência, so- 
rridente, mesmo tendo uma deficiência. Tal como descrito por Jede e Spuldaro (2009) a deficiência foi encarada por estes idosos como uma condição que acarreta alterações na autoimagem e acentua os sentimentos de fragilidade, dependência e insegurança. De acordo com Costa, Melo, Garanhani e Fujisawa (2010), é comum as pessoas com deficiência experimentarem sentimentos de vergonha e inferioridade, uma vez que convivem com o estigma diante da deficiência. O corpo passa a ser visto como objeto de vergonha, já que adquirem incapacidades em relação ao padrão vigente de produtividade da sociedade.

Em função da dependência funcional destes idosos, o significado da dependência para estes indivíduos e seus familiares se produz em uma maior ou menor tolerância e aceitação e proporciona melhor ou pior suporte instrumental, informativo, material e afetivo. Predomina, assim, um padrão de interação que reforça os comportamentos dependentes, aumentando as suas frequências (Neri, 2006). Para Baltes (1996), essa dependência significa perdas, com dificuldades nas funcionalidades físicas e psicossociais, mas também ganhos, com relação à obtenção de atenção, contato social e controle passivo, sendo uma estratégia adaptativa.

É interessante observar, ainda, que a figura 1 refere-se a uma senhora sorridente apoiada em uma bengala. A constituição da amostra foi de maioria do sexo masculino e mesmo assim, na escolha da foto, parece não ter sido influenciada pelo fator sexo (feminino), os resultados sugerem que, a prevalência na escolha e suas representações sociais foram o estado de espírito e a alegria da pessoa da foto.

\section{Importância da família}

Mesmo tendo-se verificado alterações na autoimagem dos participantes, foi possível identificar, a partir da escolha das fotos 4 e 5 , um certo senso de ajustamento psicológico (Resende \& Neri, 2009).
Os motivos relatados desta escolha foram devidos às fotos representarem "companheirismo e apoio para com a pessoa com deficiência", principalmente por parte da família.

Segundo Dorsett (2001), o ajustamento psicológico é um processo dinâmico. Apesar de não ser consensual na literatura quais os elementos fundamentais para alcançá-lo após uma deficiência física, os mais aceitos são segurança financeira, nível educacional ou socioeconômico mais alto, acessibilidade ambiental, idade menos avançada quando da aquisição da deficiência, a condição de casado e rede de relações apoiadora.

Assim, pode-se perceber neste grupo de idosos, a prevalência de alguns destes elementos, que se revelaram importantes fatores de proteção, tais como certo nível instrucional, a condição de casado, nível econômico estável, religiosidade e moradia própria e na companhia de familiares. Tais variáveis podem funcionar como recursos facilitadores dos resultados positivos encontrados para o bem-estar subjetivo das pessoas com deficiência física (Caprara \& Steca, 2005).

Nogueira (2001) refere que o apoio social a pessoas com deficiência pode tornar-se mais significativo, pois é capaz de conferir ao indivíduo, o sentimento de ser amado e estimado, bem como desperta uma sensação de controle sobre sua própria vida. Este quadro pode remeter ao conceito de autonomia, entendida por Ryff (1989) como a autodeterminação e independência, capacidade de tomar decisões sem ser influenciado por pressões sociais, mesmo diante da necessidade de auxílio para desempenhar suas atividades de vida diária ou suas escolhas.

Em contraposição ao conceito de autonomia, Batista et al. (2008) definem a dependência a partir de um conjunto de situações que configurem incapacidade física, psíquica ou intelectual que tornem os idosos frágeis, necessitando do auxílio de outrem para a realização das atividades de vida diária. Farias e Buchalla (2005) assumem que o 
senso de autonomia torna-se mais útil que a independência, como um objetivo global, uma vez que é possível restaurá-la por completo, mesmo quando o indivíduo continua com dependência.

Algumas representações dos participantes exemplificam este aspecto:

"Já anda em uma cadeira de roda, como eu comecei, e acompanhado com a mulher, como eu" (E 3, M, 73 a).

"É o meu dia-a-dia,..., no dia estou sentado na cadeira, olhando o tempo, assistindo tv, tendo contato com as pessoas. Sou feliz assim, tenho minha esposa, esse aqui é meu neto que me ajuda muito..." (E 5, M, 61 a).

"Eu gostei, assim, a mulher tá conversando com ele, como quem tá dando uma palavra de conforto" (E 12, F, 61 a).

Nos discursos dos entrevistados, pode-se observar a importância atribuída à relação com o outro, como o companheiro (a). A condição de casado pode estar relacionada à percepção de uma melhor qualidade de vida e aos arranjos de vida mais independentes. Ademais, a relação conjugal oferece maior disponibilidade de ajuda instrumental e apoio social (Caprara \& Steca, 2005). Com relação à foto 5 , destaca-se o companheirismo como sendo a representação social da dependência, ou seja, a importância da presença de alguém ao lado da pessoa com deficiência, já citada anteriormente na foto 4. De acordo com estes idosos, é importante e imprescindível o apoio e a ajuda de alguém, principalmente da família.

Essa foto, para alguns, representa tanto um familiar que está em cadeira de rodas, como a si próprio, sendo ilustrado nos relatos abaixo:

"A mulher acompanha a pessoa com cadeira de roda, tem a família, isso é muito importante..." (E 3, M, 73 a).

"É dela ser de cadeira de rodas, que eu tinha um neto de cadeira de rodas e ele morreu há dois anos. E lembrei dele, eu que cuidava dele" (E 8, F, 70 a).

"Pareceu comigo sentado nessa cadeira, a moça de lado, que é minha filha" (E 4, M, 63 a).

\section{Perspectiva futura positiva}

A foto 2 representa, segundo os entrevistados, "a alegria, a simpatia, o fato da pessoa estar de pé, não estando numa cadeira de rodas", representando a forma como alguns pretendem se ver daqui há algum tempo, tendo esperança em suas reabilitações associada ao tratamento fisioterápico. Para alguns, o fato de estar sorrindo, estar com uma boa postura, faz com que não pareça que a pessoa da foto possua uma deficiência, pois a imagem da pessoa aparenta estar sadia.

“... é uma pessoa deficiente, mas tá com uma boa postura, tá feliz, sorridente” (E 9, F, 67 a).

Os próprios participantes vivenciavam a situação de impossibilidade de andar, mas ao contrário deste pensamento, expressaram sentimentos positivos, enfatizando a esperança da reabilitação como meta a ser atingida.

"É como eu pretendo me ver daqui a uns determinados dias. Procuro dá menos ocupação a minha esposa, tô conseguindo fazer barba, cortar unha, já tô me sentindo um progresso" (E 5, M, 61 a).

O relato acima vai ao encontro do que preconiza a teoria da dependência aprendida (Neri, 2006), no sentido de que quando o comportamento independente é reforçado, ocorre um aumento de sua frequência e, consequentemente, das condições que favorecem à sua independência.

A foto com menor percentagem de escolha pelos participantes da pesquisa foi a 3 . A foto representa uma menina deficiente dançando numa cadeira de rodas. Os poucos que a escolheram, fizeram porque "acharam que a menina, mesmo tendo uma deficiência, estava numa situação boa, de alegria, divertimento".

"Tá melhor, numa situação boa, chega de tristeza, a vida já é uma tristeza" (E 2, M, 69 a).

"Porque tem mais divertimento, gostei mais do jeito dela" (E 13, F, 81 a).

Diante disto, pode-se apreender que as RS dos participantes estiveram ancoradas em alterações da autoimagem, companheirismo da família e es- 
perança na reabilitação, de aos poucos voltarem a andar e reconquistar suas independências. As expectativas positivas podem ser compreendidas na medida em que se tratava de pessoas que estavam em processo de reabilitação fisioterápica e dispunham de uma série de condições que favorecia a esperança da recuperação.

Por conseguinte, não poder andar não implica essencialmente em estar impedido de pensar, decidir e desejar. A dependência não pode ser analisada como uma perda de autonomia, e a autoestima é um sentimento a ser alimentado permanentemente, desde que a pessoa encontre, em seu meio, condições para conhecer-se e aceitar-se, mesmo com algumas limitações (Sanchez, 2000). Desse modo, envelhecer com uma deficiência física, é um processo que exige competência adaptativa e resiliência aos eventos de vida e aos desafios acarretados pela deficiência (Resende \& Neri, 2009).

Para ampliar a discussão é necessário ressaltar, conforme Resende e Gouveia (2009), que viver e envelhecer implicam alterações gradativas nos domínios físico, psicológico, social e ambiental. Entretanto, é inegável o preconceito contra o envelhecimento e a negação da sociedade quanto a esse fenômeno, o que colabora para a dificuldade de se pensar políticas específicas para esse grupo (Andrade, Sena, Pinheiro, Meira, \& Lira, 2013), demonstrando o despreparo para acolher e oferecer suporte a todos que apresentam algum tipo de limitação funcional (Areosa \& Areosa, 2008).

\section{Considerações Finais}

Esta pesquisa teve o objetivo de conhecer as concepções sobre o fenômeno da deficiência física de pessoas idosas, analisando por meio dos discursos, a representação social e o sentido da deficiência física.

Pode-se constatar que há uma dependência física e psíquica por parte dos idosos em relação a seus familiares e/ou pessoas próximas, devido ao estado em que se encontram, por estarem impos- sibilitados de desenvolver algumas atividades do dia-a-dia, por não conseguirem andar normalmente, não poderem movimentar-se e pela necessidade de um maior afeto e carinho.

Ao mesmo tempo foi possível verificar, mediante os discursos dos entrevistados, a esperança da reabilitação, vislumbrando um futuro a ser vivido de forma autônoma e independente. A dependência, segundo estes idosos, é "dar trabalho" para o cuidador, por isso pensam em melhorar para tornarem-se independentes. Ao mesmo tempo, acreditam na importância de se ter alguém da família para acompanhá-los, ajudando-os no que precisam, obtendo-se assim, maior apoio e bem-estar.

Enfim, o ser dependente para os participantes desta pesquisa, significa um período de transição em que eles acreditam na dinâmica da reabilitação, prevalecendo um forte desejo de recuperação total da deficiência. Fatores como a motivação para o estabelecimento de metas, a manutenção de uma vida ativa, produtiva e saudável e o cultivo da espiritualidade, devem ajudar no estado geral do idoso, conferindo condições essenciais ao processo de reabilitação.

O estudo multidisciplinar sobre o envelhecimento com uma deficiência é uma área nova e emergente, tanto no campo da Gerontologia como no das Ciências Sociais e da Saúde, e muito ainda precisa ser investigado sobre a relação entre deficiência e envelhecimento.

Acredita-se que, esta pesquisa, possa fornecer contribuições para discussão e reflexão do tema "envelhecimento com dependência" como um desafio a ser incorporado pelo campo da Saúde Pública no intuito de que sejam promovidas e implantadas políticas públicas de saúde e psicossociais que primem por um envelhecimento autônomo e independente.

\section{Referências}

Albrecht, G. L., \& Devlieger, P. J. (1999). The disability paradox: high quality of life against all odds. 
Social Science \& Medicine, 48(8), 977-988. doi: 10.1016/S0277-9536(98)00411-0

Andrade, L. M., Sena, E. L. da S., Pinheiro, G. M. L., Meira, E. C., \& Lira, L. S. S. P. (2013). Public policies for the elderly in Brazil: an integrative review. Ciência e Saúde Coletiva, 18(12), 3543-3552.

Araújo, L., Sá, E. C. N., \& Amaral, E. B. (2011). Corpo e velhice: um estudo das representações sociais entre homens idosos. Psicologia: Ciência e Profissão, 31(3), 468-481.

Areosa, S. V. C., \& Areosa, A. L. (2008). Envelhecimento e dependência: desafios a serem enfrentados. Textos \& Contextos, 7(1), 138-150.

Baltes, M. (1996). The many faces of dependency in old age. New York: Cambridge University Press.

Baltes P. B. (1987). Theoretical propositions of the life span developmental psychology: On the dynamics between growth and decline. Developmental Psychology, 23, 611-696. doi: 10.1037/0012-1649.23.5.611

Baltes P. B., \& Baltes M. M. (1990). Psychological perspectives on successful aging: The model of selective optimization with compensation. In: Baltes P. B, Baltes M. M. (Eds), Successful Aging. Perspectives from Behavioral Sciences (pp. 1-34). New York: Cambridge University Press.

Banco Mundial. (2011). Envelhecendo em um Brasil mais velho. Washington DC: Banco Mundial. Recuperado de http://siteresources. worldbank.org/BRAZILINPOREXTN/Resources/3817166-1302102548192/Envelhecendo Brasil_Sumario_Executivo.pdf

Bardin, L. (2008). Análise de conteúdo. (19 ed.). Lisboa: Edições 70.

Barduzzi, G. de O., Rocha Júnior, R. P., Sousa Neto, de J. C., \& Aveiro, M. C. (2013). Functional capacity of elderly with osteoarthritis who undergone to aquatic and land physical therapy. Fisioterapia em Movimento, 26(2), 349-360. doi: 10.1590/S0103-51502013000200012
Batista, A. S., Jaccoud, L. de B., Aquino, L., \& ElMoor, P. D. (2008). Envelhecimento e dependência: desafios para a organização da proteção social. Brasília: MPS, SPPS.

Brasil. (2003). Estatuto do idoso. Brasília: Senado Federal.

Brasil. (2012). Ministério da Saúde. Conselho Nacional de Saúde.

Caprara, G. V., \& Steca, P. (2005). Affective and social selfregulatory efficacy beliefs as determinants of positive thinking and happiness. European Psychologist, 10(4), 275-286. doi: 10.1027/1016-9040.10.4.275

Carstensen, L. L. (1992). Socio emotional selectivity theory: Social activity in life-span context. Psychology and Aging, 11, 195-217.

Castanha, A. R., \& Araújo, L. F. (2006). Álcool e agentes comunitários de saúde: um estudo das representações sociais. Psico-USF, 11(1), 85-94.

Costa, V. de S. P., Melo, M., Garanhani, M. L., \& Fujisawa, D. S. (2010). Representações sociais da cadeira de rodas para a pessoa com lesão da medula espinhal. Revista Latino-Americana de Enfermagem, 18(4), 1-8.

Coutinho, M. da P. de L., Estevam, I. D., Araújo, L. F. de, \& Araújo, L. S. (2011). Prática de privação de liberdade em adolescentes: um enfoque psicossociológico. Psicologia em Estudo, 16(1), 101-109.

Dorsett, P. A. (2001). Spinalcordinjury: how do people cope? (Tese de Doutorado Não-Publicada, University of Queensland, Australia).

Farias N., \& Buchalla, O. M. (2005). Classificação internacional de funcionalidade, incapacidade e saúde da Organização Mundial da Saúde: conceitos, usos e perspectivas. Revista Brasileira de Epidemiologia, 8(2), 187-93. doi: 10.1590/ S1415-790X2005000200011

Fjell, A. M., Westlye, L. T., Grydeland, H., Amlien, I., Espeseth, T., \& Reinvang, I. (2013). Alzheimer Disease Neuroimaging Initiative. Critical ages in the life course of the adult brain: nonlinear subcortical aging. Neurobiology of aging, 
34(10), 2239-2247. doi:10.1016/j.neurobiolaging.2013.04.006

Instituto Brasileiro de Geografia e Estatística (IBGE, 2010). Características gerais da população, religião e pessoas com deficiência. Río de Janeiro. Recuperado de http://www.ibge.gov.br/home/ estatistica/populacao/censo2010/caracteristicas_religiao_deficiencia/default_caracteristicas_religiao_deficiencia.shtm

Jede, M., \& Spuldaro, M. (2009). Cuidado do idoso dependente no contexto familiar: uma revisão de literatura. RBCEH, 6, 413-421.

Justo J. S., \& Vasconcelos, M. S. (2009). Pensando a fotografia na pesquisa qualitativa em psicologia. Estudos e Pesquisas em Psicologia, 9(3), 760-774.

Moraes, E. N. de, Moraes, F. L. de, \& Lima, S. P. P. (2010). Características biológicas e psicológicas do envelhecimento. Revista Médica de Minas Gerais, 20(1), 67-73.

Moscovici, S. (2009). Representações sociais: investigações em psicologia social (6 $6^{\mathrm{a}}$ ed.). Petrópolis, RJ: Vozes.

Neiva-Silva, L., \& Koller, S. H. (2002). O uso da fotografia na pesquisa em Psicologia. Estudos de Psicologia, 7(2), 237-250.

Neri, A. L. (1991). Envelhecer num país de jovens: significados de velho e velhice segundo brasileiros não idosos. Campinas, SP: UNICAMP.

Neri, A. L. (2000). A formação de recursos humanos em gerontologia: o papel da pós-graduação. Arquivos de Geriatria e Gerontologia, 4(3), 69-104.

Neri, A. L. (2004). O que a Psicologia tem a oferecer ao estudo e à intervenção no campo do envelhecimento no Brasil, hoje. In A. L. Neri \& M. S. Yassuda, Velhice bem-sucedida (pp. 13-27). Campinas, SP: Papirus.

Neri, A. L. (2006). Teorias psicológicas do envelhecimento: percurso histórico e teorias atuais. In E. V. Freitas, L. Py, F. A. X. Cançado, J. Doll, \& M. L. Gorzoni (Eds) (2006). Tratado de
Geriatria e Gerontologia, (pp. 58-77). Rio de Janeiro: Guanabara.

Neri, A. L., \& Jorge, M. D. (2006). Atitudes e conhecimentos em relação à velhice em estudantes de graduação em educação e em saúde: subsídios ao planejamento curricular. Estudos de Psicologia, 23(2), 127-137.

Nóbrega, S. M., \& Coutinho, M. P. L. (2003). O teste de associação livre de palavras. In M. P. L. Coutinho e cols. (Org.). Representações sociais: Abordagem interdisciplinar (pp. 67-77). João Pessoa, PB: UFPB.

Nogueira, E. J. (2001). Rede de relações sociais: Um estudo transversal com homens e mulheres pertencentes a três grupos etários. (Tese de Doutorado não-publicada, UniversidadeEstadual de Campinas, SP).

Organização das Nações Unidas (ONU, 2010). World Population Prospects: the 2010 revision. New York: Unites Nations.

Organização Mundial de Saúde (OMS, 1984). The uses of epidemiology in the study of the elderly. New Hope: Geneva: Geneva.

Organização Mundial de Saúde (OMS, 2010). World Health Report 2010. Suíça: OMS.

Resende, M. C. de, \& Gouveia, V. V. (2011). Quality of life in adults with physical disability. Psicologia: Reflexão e Crítica, 24(1), 99-106.

Resende, M. C. de, \& Neri, A. L. (2005). Atitudes de adultos com deficiência física frente ao idoso, à velhice pessoal e a pessoas com deficiência física. Estudos de Psicologia, 22,123-131.

Resende, M. C. de, \& Neri, A. L. (2009). Ajustamento psicológico e perspectiva de velhice pessoal em adultos com deficiência física. Psicologia em Estudo, 14(4), 767-776.

Resende, M. C, \& Rabelo, D. F. (2004). Envelhecer com Deficiência Física: Experiência Com Grupos Educativos. Estudos interdisciplinares sobre o envelhecimento, 6, 41-51.

Resolução $n^{\circ}$ 466, de 12 de dezembro de 2012. Dispõe sobre diretrizes e normas regulamentado- 
ras de pesquisas envolvendo seres humanos. Recuperado de http://conselho.saude.gov.br/ resolucoes/2012/Reso466.pdf

Ryff, C.D. (1989). Happiness is everything, or is it? Explorations on the meaning of psychological well-being. Journal of Personality and Social Psychology, 57, 1069-1081. doi: 10.1037/00223514.57.6.1069
Sanchez, M. A. S. (2000). A dependência e suas implicações para a perda de autonomia: estudo das representações para idosos de uma unidade ambulatorial geriátrica. UNATI, 3(3), 1-8.

Saraiva, E. R. A., \& Coutinho, M. P. L. (2012). A difusão da violência contra idosos: um olhar psicossocial. Psicologia e sociedade, 24, 112-121. 
